\title{
Special issue on bio-micro/nano manipulation and mechatronics -from International Symposium on Micro-Nanomechatronics and Human Science
}

\author{
Masahiro Nakajima • Tatsuo Arai
}

Published online: 20 November 2012

(C) Springer-Verlag Berlin Heidelberg 2012

Micro/Nano mechatronics have been used in our daily devices or products to achieve miniaturization, low-energy consumption, low-cost, minimum material usage, high-efficiency, high-integration, and high-functionality. One of the significant applications has been investigated based on the micro/nano mechatronics in biological and medical fields. To understand and to control biological system, micro-nano manipulation is one of the key technologies for innovations from engineering and science viewpoints.

This special issue is basically organized by the selected papers which were presented in the International Symposium on Micro-Nanomechatronics and Human Science (MHS) from 2005 to 2010. The symposiums were held as joined with the symposium on "System Cell Engineering by Multi-scale Manipulation" which is the Scientific Research of Priority Areas, MEXT, Japan. Hence, this special issue shows the cutting edges of biological applications by micro/nano manipulation and mechatronics technologies including current and future applications of this research fields.

As brief introductions of each paper are follows,

Issue 1: The paper by Jaehoon Jung et al., entitled "Microchip device for measurement of body volume of C. elegans as bioindicator application" presented a micro-fluidic device to analyze a Caenorhabditis elegans (C. elegans) as bioindicator application using capacitance sensor. Next, the paper by Chanh-Nghiem Nguyen et al. entitled "Real-time Precise 3D Measurement of Micro Transparent Objects Using All-In-Focus Imaging

M. Nakajima $(\bowtie)$

Nagoya University,

Nagoya, Japan

e-mail: nakajima@mein.nagoya-u.ac.jp

T. Arai

Osaka University,

Suita City, Japan
System" presented a All-In-Focus imaging system to obtain 3D position measurement of both the micromanipulator and target micro object for cell manipulation application. Next, the paper by Yoshitake Akiyama et al. entitled "Evaluation of mechanical stimulation effect on cellular orientation under confluence based on 2D-FFT and principal component analysis" presented the evaluation of mechanical stimulation for a muscular cell sheet by the combination of two-dimensional fast Fourier transform (2D-FFT) and principal component analysis.

Issue 2: The paper by Kenji Inoue et al. entitled "Micromanipulation Using Micro Hand with Two Rotational Fingers" presented an 8-DOF micro hand consisting of two rotational fingers to handle a microobject with rotational motion of about its two axes by virtual stick method. Next, the paper by Nam Cao Hoai Le et al. entitled "Highly-sensitive fluorescence detection and imaging with microfabricated total internal reflection (TIR)-based devices" presented the total internal reflection (TIR)-based devices for highly-sensitive and high-resolution fluorescence imaging fabricated by micro electro mechanical systems (MEMS) fabrication technology. Next, the paper by Masayasu Suzuki et al. entitled "Detection and collection system of target single cell based on respiration activity" presented the systematic technique for detection and collection of single cell respiration activity by the microarrayed optical oxygen sensor.

Issue 3: The paper by Kenichi Ohara et al. entitled "Dextrous cell diagnosis using two-fingered microhand with micro force sensor" presented the measurement of physical cell property using a two-fingered micro hand system with a micro force sensor. Next, the paper by Shuichi Yamaguchi et al. entitled "Stable 
ejection of micro droplets containing microbeads by a piezoelectric inkjet head" presented the experimental evaluation of stable ejection of micro-particles by the nozzle diameter, the particle diameter, and the ejection waveform. Next, the paper by Tomonori Kano et al. entitled "A microfluidic device for bacteria immobilization in a microporous carrier by dielectrophoresis" presented the immobilization of bacteria in micropores is demonstrated using positive dielectrophoresis (DEP) in a microfluidic device for an efficient bacteria screening platform.
We would like to express our sincere appreciations to the Editors-in-Chiefs of the Journal of Micro-Nano Mechatronics, Prof. Paolo Dario, Prof. Toshio Fukuda and Prof. Metin Sitti for giving us the opportunity to prepare this special issue. We would like to sincere gratitude to all supporting people for publication, especially, Dr. Thomas Ditzinger, Dr. Edoardo Sinibaldi, and Dr. Kenichi Ohara. We are deeply indebted to all reviewers for their professional efforts and hard works. Finally, we are grateful to all authors for their contributions, without which this special issue could not have been published. 